\title{
Throat quantization of the Schwarzschild-Tangherlini(-AdS) black hole
}

\author{
Hideki Maeda ${ }^{1, \star}$ \\ ${ }^{1}$ Department of Electronics and Information Engineering, Hokkai-Gakuen University, Sapporo, Japan
}

\begin{abstract}
By the throat quantization pioneered by Louko and Mäkelä, we derive the mass and area/entropy spectra for the Schwarzschild-Tangherlini-type asymptotically flat or AdS vacuum black hole in arbitrary dimensions. Using the WKB approximation for black holes with large mass, we show that area/entropy is equally spaced for asymptotically flat black holes, while mass is equally spaced for asymptotically AdS black holes. Exact spectra can be obtained for toroidal AdS black holes in arbitrary dimensions including the three-dimensional BTZ black hole.
\end{abstract}

\section{Classical vacuum solution with maximally symmetric base manifold}

Let us consider the vacuum Einstein equations with a cosmological constant

$$
\mathcal{R}_{\mu \nu}-\frac{1}{2} g_{\mu \nu} \mathcal{R}+\Lambda g_{\mu \nu}=0
$$

for the $n(\geq 3)$-dimensional spacetime with spherical ( $k=$ $1)$, planar $(k=0)$, or hyperbolic $(k=-1)$ symmetry $\left(\mathcal{M}^{n}, g_{\mu \nu}\right) \approx\left(M^{2}, g_{A B}\right) \times\left(K^{n-2}, \gamma_{a b}\right)$ with the general metric

$$
\begin{aligned}
\mathrm{d} s^{2} & =g_{\mu \nu}(x) \mathrm{d} x^{\mu} \mathrm{d} x^{\nu} \\
& =g_{A B}(\bar{y}) \mathrm{d} \bar{y}^{A} \mathrm{~d} \bar{y}^{B}+R(\bar{y})^{2} \gamma_{a b}(z) \mathrm{d} z^{a} \mathrm{~d} z^{b},
\end{aligned}
$$

where $g_{A B}$ is an arbitrary two-dimensional Lorentzian metric on $M^{2}$ and $\gamma_{a b}$ is the metric on the $(n-2)$-dimensional maximally symmetric space $K^{n-2}$ with its curvature $k$. In three dimensions $(n=3), K^{n-2}$ is one-dimensional which corresponds to $k=0$.

By the Birkhoff's theorem, the most general solution with non-constant areal radius $R$ is the following:

$$
\begin{aligned}
& \mathrm{d} s^{2}=-f(\mathbf{m}, r) \mathrm{d} T^{2}+f(\mathbf{m}, r)^{-1} \mathrm{~d} r^{2}+r^{2} \gamma_{a b} \mathrm{~d} z^{a} \mathrm{~d} z^{b}, \\
& f(\mathbf{m}, r):=k-\frac{2 \kappa_{n}^{2} \mathbf{m}}{(n-2) V_{n-2}^{(k)} r^{n-3}}+\frac{r^{2}}{l^{2}},
\end{aligned}
$$

where $\kappa_{n}^{2}:=8 \pi G_{n}$, defined by the $n$-dimensional Newton constant $G_{n}$, and $l:=\sqrt{-(n-1)(n-2) / 2 \Lambda}$ is the AdS radius. This is the topological generalization of the Schwarzshild-Tangherlini solution in the presence of a cosmological constant. $\mathbf{m}$ is the mass parameter and its range that gives a black-hole configuration is summarized in Table 1. The global structures of the resulting black hole with a single horizon for $\Lambda \leq 0$ are drawn in Fig. 1 . Hereafter we assume that $K^{n-2}$ is compact and then $V_{n-2}^{(k)}$ represents the surface area of the submanifold $K^{n-2}$.
Table 1. The range of $\mathbf{m}$ for the black-hole configuration given by the Schwarzshild-Tangherlini-type solution (3), where $\mathbf{m}_{\mathrm{ex}}$ is the mass for the extremal horizon.

\begin{tabular}{cccc}
\hline \hline & $k=1$ & $k=0$ & $k=-1$ \\
\hline$\Lambda=0$ & $\mathbf{m}>0$ & None & None \\
\hline$\Lambda<0$ & $\mathbf{m}>0$ & $\mathbf{m}>0$ & $\mathbf{m} \geq \mathbf{m}_{\mathrm{ex}}(<0)$ \\
\hline \hline
\end{tabular}

In order to investigate the quantum aspects of this Schwarzshild-Tangherlini-type black hole, one needs to quantize the Einstein equations (1). The ADM formalism of the Einstein equations shows that general relativity is a dynamically constrained system. Reduced phase-space quantization is one possible way to quantize such a system, in which one performs canonical quantization on the constraint surface after solving all the (classical) constraint equations.

Of course, it is hopeless to accomplish this with full generality. However, it is possible in the midisuperspace approach for the symmetric spacetime (2). Throat quantization pioneered by Louko and Mäkelä [1] is the reduced phase-space quantization for the spacetime (2) with a certain set of canonical variables.

\section{Reduced phase space}

In order to write all the classical constraint equations in solvable forms, one carefully chooses the canonical variables. This is obvious when we choose the following ADM variables for the metric:

$$
\begin{aligned}
\mathrm{d} s^{2}= & -N(t, r)^{2} \mathrm{~d} t^{2}+\bar{\Lambda}(t, r)^{2}\left(\mathrm{~d} r+N_{r}(t, r) \mathrm{d} t\right)^{2} \\
& +R(t, r)^{2} \gamma_{a b} \mathrm{~d} z^{a} \mathrm{~d} z^{b} .
\end{aligned}
$$

Integrating over $K^{n-2}$, the Einstein-Hilbert action reduces to the following two-dimensional ADM form:

$$
I_{\mathrm{ADM}}=\int \mathrm{d} t \int \mathrm{d} r\left(\dot{\bar{\Lambda}} P_{\bar{\Lambda}}+\dot{R} P_{R}-N H-N_{r} H_{r}\right),
$$


(a)

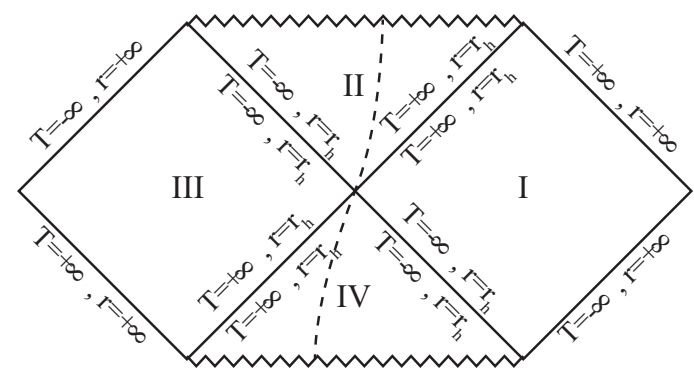

(b)

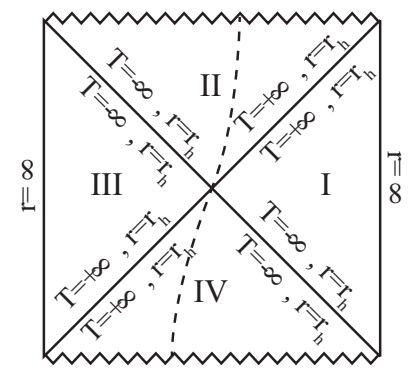

Figure 1. Penrose diagrams of the Schwarzschild-Tangherlinitype black hole (3) with a single Killing horizon for (a) $\Lambda=0$ and (b) $\Lambda<0$. A dashed curve represents a constant $T$ timelike hypersurface inside the Killing horizon.

where a dot denotes the derivative with respect to $t$. While $\bar{\Lambda}$ and $R$ are dynamical variables with their momentum conjugates $P_{\bar{\Lambda}}$ and $P_{R}, N$ and $N_{r}$ are Lagrange multipliers providing the constraint equations $H=0$ ansd $H_{r}=0$, respectively. These constraint equations cannot be solved because the super-Hamiltonian $H$ and super-momentum $H_{r}$ are very complicated functionals of $\bar{\Lambda}, R, P_{\bar{\Lambda}}$, and $P_{R}$ in this ADM formalism.

Thus, in order to solve the constraint equations, one has to find a nice set of the canonical variables which are given by a canonical transformation from the ADM variables $\left\{\bar{\Lambda}, P_{\bar{\Lambda}} ; R, P_{R}\right\}$. In the seminal paper [2], Kuchař introduced a set of geometrodynamical variables to solve constraint equations and obtain the reduced phase-space for general spherically symmetric spacetime in four dimensions $(n=4)$ without a cosmological constant $(\Lambda=$ $0)$. In this section, we review his method for $n(\geq 4)$ dimensional spherically symmetric spacetime $(k=1)$.

Kuchar showed that the transformation $\left\{\bar{\Lambda}, P_{\bar{\Lambda}} ; R, P_{R}\right\} \rightarrow\left\{M, P_{M} ; S, P_{S}\right\}$ defined by

$$
\begin{aligned}
S:=R, \quad P_{S}:=P_{R}-\frac{1}{R^{\prime}}\left(\bar{\Lambda} P_{\bar{\Lambda}}^{\prime}+P_{M} M^{\prime}\right), \\
M:=\frac{(n-2) V_{n-2}^{(1)}}{2 \kappa_{n}^{2}} R^{n-3}(1-F), \quad P_{M}:=-\frac{y \bar{\Lambda}}{F}
\end{aligned}
$$

is canonical, where a prime denotes the derivative with respect to $r$ and $y$ and $F$ are defined by

$$
y:=N^{-1}\left(\dot{R}-N_{r} R^{\prime}\right), \quad F:=-y^{2}+\bar{\Lambda}^{-2} R^{\prime 2} .
$$

The new canonical variables have clear physical/geometrical meanings; $M$ is the Misner-Sharp quasi-local mass [3] and $S$ is the areal radius. In order to show the canonicality of the transformation, the following boundary conditions are required:

$$
\begin{array}{ll}
N \simeq N_{\infty}(t)+O\left(r^{-\epsilon_{1}}\right), & N_{r} \simeq N_{r}^{\infty}(t) r^{-(n-3) / 2-\epsilon_{2}}, \\
\bar{\Lambda} \simeq 1+\bar{\Lambda}_{1}(t) r^{-(n-3)}, & R \simeq r+R_{1}(t) r^{-(n-4)-\epsilon_{4}},
\end{array}
$$

where $\epsilon_{1}$ is a positive number and $\epsilon_{2}$ and $\epsilon_{4}$ satisfy $\epsilon_{2}>$ $\max [0,-(n-5) / 2]$ and $\epsilon_{4}>\max [0,-(n-5)]$.

The new action after the canonical transformation with the boundary term is given by

$$
\begin{aligned}
I_{\text {geo }}= & \int \mathrm{d} t \int \mathrm{d} r\left(P_{M} \dot{M}+P_{S} \dot{S}-N \bar{H}-N_{r} \bar{H}_{r}\right) \\
& -\int \mathrm{d} t\left(N_{+} M_{\infty}-N_{-} M_{\infty}\right),
\end{aligned}
$$

where the second term is the boundary term and

$$
\begin{aligned}
& \bar{H}:=\frac{\left(F+y^{2}\right) P_{M} M^{\prime}}{y S^{\prime}}+y P_{S}, \\
& \bar{H}_{r}:=P_{M} M^{\prime}+P_{S} S^{\prime}, \\
& M_{\infty}(t):=\lim _{r \rightarrow \pm \infty} M, \\
& N_{ \pm}(t):= \pm \lim _{r \rightarrow \pm \infty} N .
\end{aligned}
$$

In terms of the new variables, $F$ and $y$ are given by

$$
\begin{aligned}
F & =1-\frac{2 \kappa_{n}^{2} M}{(n-2) V_{n-2}^{(1)} S^{n-3}}, \\
y^{2} & =\frac{F^{3} P_{M}^{2}}{S^{\prime 2}-F^{2} P_{M}^{2}} .
\end{aligned}
$$

The constraint equations $\bar{H}=0$ and $\bar{H}_{r}=0$ in terms of the new variables reduce to $M^{\prime}=0$ and $P_{S}=0$, of which solution is simply $M=\mathbf{m}(t)$ and $P_{S}=0$.

Then, the spatial integration in the new action (12) can be easily performed on the constraint surface to give the following Kuchař's one-dimensional reduced action:

$$
I_{\mathrm{K}}[\mathbf{m}, \mathbf{p}]=\int \mathrm{d} t\left[\mathbf{p}(t) \dot{\mathbf{m}}(t)-\left(N_{+}-N_{-}\right) \mathbf{m}(t)\right]
$$

where $\mathbf{p}:=\int_{-\infty}^{\infty} P_{M}(r, t) \mathrm{d} r$. The reduced action (19) depends only $\mathbf{m}(t)$ and its conjugate p. $N_{ \pm}(t)$, the values of the lapse $N$ at either ends of the spatial slice, are prescribed functions and not varied. The Hamilton's equation for $\mathbf{m}$ is simply $\dot{\mathbf{m}}=0$, whose solution requires $\mathbf{m}=$ constant, which is equal to the ADM mass. This is a different realization of the Birkhoff's theorem, showing that $\mathbf{m}$ carries all the information about the local geometry of the classical solution.

As shown in [4], this Kuchař reduction to the onedimensional action (19) can be achieved for general $k$ with a negative cosmological constant. In this case, $M$ is replaced by the following generalized Misner-Sharp mass [5]:

$$
M=\frac{(n-2) V_{n-2}^{(k)}}{2 \kappa_{n}^{2}} R^{n-3}\left\{-\frac{R^{2}}{l^{2}}+(1-F)\right\} .
$$




\section{Throat quantization}

Since the reduced action (19) does not contain any information that the spacetime represents a black hole, canonical quantization of this system does not provide quantum mechanics of the black hole given by Eq. (3). For this purpose, one therefore needs another canonical transformation from the Kuchař action (19). This is what Louko and Mäkelä did for the four-dimensional spherically symmetric spacetime without a cosmological constant [1]. In [6], we generalized their result in the case of general $k$ with $\Lambda(\leq 0)$ in arbitrary dimensions. We summarize our result in this section.

\subsection{Reduced action with throat variables}

Starting from the Kuchař reduce action (19), we first set $N_{+}=1$ and $N_{-}=0$. This is a gauge choice at spatial infinities. $N_{+}=1$ means that $t$ coincides with the proper time for an observer at rest at the right-hand spacelike infinity, up to the additive constant. (See Fig. 1.) On the other hand, $N_{-}=0$ means that the hypersurface at the left-hand asymptotic infinity is frozen for all $t$. Thus, the physical meaning of the time $t$ is uniquely deterimined as a proper time of the static observer at one spacelike infinity. Now the action becomes $I_{\mathrm{K}}=\int \mathrm{d} t(\mathbf{p} \dot{\mathbf{m}}-\mathbf{m})$, for which the Hamiltonian is simply $\mathbf{m}$, the generalized Misner-Sharp mass.

Following Louko and Mäkelä, we take a canonical transformation $(\mathbf{m}, \mathbf{p}) \rightarrow\left(a, p_{a}\right)$ from this action, where the relations between old and new variables are given by

$$
\begin{aligned}
& |\mathbf{p}|=\int_{a}^{a_{\mathrm{h}}(\mathbf{m})} \frac{\mathrm{d} b}{\sqrt{-f(\mathbf{m}, b)}}, \\
& p_{a}=\operatorname{sgn}(\mathbf{p}) \sqrt{-\left(\frac{(n-2) V_{n-2}^{(k)}}{\kappa_{n}^{2}}\right)^{2} a^{2(n-3)} f(\mathbf{m}, a),}
\end{aligned}
$$

where the function $f$ is defined by Eq. (4) and $a_{\mathrm{h}}$ is defined by $f\left(\mathbf{m}, a_{\mathrm{h}}\right)=0$. This is a time-independent canonical transformation and therefore the Hamiltonian $H\left(a, p_{a}\right)$ for the new set of variable $\left(a, p_{a}\right)$ is simply $\mathbf{m}\left(a, p_{a}\right)$. The resulting new action is

$$
I_{\mathrm{LM}}=\int \mathrm{d} t\left(p_{a} \dot{a}-H\left(a, p_{a}\right)\right),
$$

where the new Hamiltonian $H\left(a, p_{a}\right)(=\mathbf{m})$ is given by

$$
\begin{aligned}
H= & \frac{(n-2) V_{n-2}^{(k)} a^{n-3}}{2 \kappa_{n}^{2}} \\
& \times\left[\left(\frac{(n-2) V_{n-2}^{(k)}}{\kappa_{n}^{2}}\right)^{-2} p_{a}^{2} a^{-2(n-3)}+k+\frac{a^{2}}{l^{2}}\right] .
\end{aligned}
$$

The domains of $a$ and $p_{a}$ are $0 \leq a<\infty$ and $-\infty<p_{a}<$ $\infty$, respectively.

Hamilton's equations in the system (23) give

$$
\frac{\mathrm{d}^{2} a}{\mathrm{~d} t^{2}}=-\frac{(n-3) \kappa_{n}^{2} \mathbf{m}}{(n-2) V_{n-2}^{(k)} a^{n-2}}-\frac{a}{l^{2}} .
$$

Indeed, this is formally equivalent to the dynamics of areal radius $r$ at the wormhole throat in the SchwarzschildTangherlini-type black-hole spacetime (3), which is located inside the Killing horizon. This wormhole throat is defined by a radial timelike geodesic with constant $T$, of which areal radius $r=a(\tau)$ is determined by the following geodesic equation:

$$
\frac{\mathrm{d}^{2} a}{\mathrm{~d} \tau^{2}}=-\frac{(n-3) \kappa_{n}^{2} \mathbf{m}}{(n-2) V_{n-2}^{(k)} a^{n-2}}-\frac{a}{l^{2}},
$$

where $\tau$ is the proper time along the geodesic. (A dashed curve in Fig. 1 is an example of such a geodesic.) The (classical) domain of $a$ is $0 \leq a \leq r_{\mathrm{h}}$, where $a=0$ and $a=$ $r_{\mathrm{h}}$ correspond to the curvature singularity and the Killing horizon, respectively.

Equation (25) is identical to Eq. (26) with $t=\tau$. The terminology "throat quantization" comes from this physical interpretation of the dynamics represented by the action (23). However, it is noted that the time $t$ is originally the proper time for an observer at one spatial infinity, not the proper time along the wormhole throat.

\subsection{Quantization: Hamiltonian operator and the boundary condition}

Finally we canonically quantize the system (23) and perform the quantum mechanics of the SchwarzschildTangherlini-type black hole. Replacing $p_{a}$ in the Hamiltonian (24) by $\hat{p}_{a}=-i \hbar \partial / \partial a$, we obtain the Schrödinger equation $\hat{H} \Psi=E \Psi$ for the wave function $\Psi=\Psi(a)$ in the stationary state. Under the Laplace-Beltrami operatorordering, the Schrödinger equation is written as

$$
\left(-\frac{\hbar^{2}}{2 m} \frac{\partial^{2}}{\partial x^{2}}+V(x)\right) \Psi=E \Psi
$$

where $x:=a^{(n-1) / 2}$ and the effective mass $m$ and potential $V(x)$ are given by

$$
\begin{aligned}
m & \equiv \frac{4(n-2) V_{n-2}^{(k)}}{(n-1)^{2} \kappa_{n}^{2}}, \\
V(x) & \equiv \frac{(n-2) V_{n-2}^{(k)}}{2 \kappa_{n}^{2}}\left(\frac{x^{2}}{l^{2}}+k x^{2(n-3) /(n-1)}\right) .
\end{aligned}
$$

The domain of $x$ is $0 \leq x<\infty$ and hence this is the quantum mechanics on the half-line. The natural inner product consistent with the Laplace-Beltrami operatorordering is

$$
\langle\Psi \mid \Phi\rangle:=\int_{0}^{\infty} \Psi^{*} \Phi \mathrm{d} x .
$$

Since the classical Hamiltonian $H$ is the generalized Misner-Sharp mass, we identify $E$ as the mass eigenvalue of the black hole.

Actually, the Hamiltonian operator $\hat{H}$ is not selfadjoint in the square-integrable Hilbert space on the halfline $\mathcal{L}^{2}([0, \infty))$ but it admits self-adjoint extensions. This 
self-adjoint extension requires the following boundary condition at the location of the classical singularity $x=0$ :

$$
\Psi(0)+\left.L \frac{\partial \Psi}{\partial x}\right|_{x=0}=0,
$$

where $L$ is a real constant [7]. $L=0$ corresponds to the Dirichlet boundary conditions, while $L=\infty$ corresponds to the Neumann boundary condition. Other values of $L$ gives the general Robin boundary conditions. With the boundary condition (31), evolution of this quantum system is unitary $(\partial\langle\Psi \mid \Phi\rangle / \partial t=0)$.

\subsection{Mass spectrum of the black hole}

Now we show the mass spectrum $E$ of the black hole under the Dirichlet $(L=0)$ boundary condition at the origin $x=0$. While an exact spectrum can be obtained for the toroidal AdS black hole $(k=0)$ in arbitrary dimensions, one obtains the mass spectra for black holes with large mass using the WKB approximation in other cases.

The mass spectrum sharply depends on the presence of the cosmological constant. For asymptotically flat black hole $(k=1)$, the spectrum for large $E$ is

$$
\begin{aligned}
E^{(n-2) /(n-3)} \simeq( & n-2) \hbar \sqrt{\pi} N \\
& \times \frac{\Gamma\left(\frac{n-1}{2(n-3)}+\frac{1}{2}\right)}{\Gamma\left(\frac{n-1}{2(n-3)}\right)}\left(\frac{(n-2) V_{n-2}^{(1)}}{2 \kappa_{n}^{2}}\right)^{1 /(n-3)},
\end{aligned}
$$

where $N$ is a large integer. By the following classical relation between the mass $E$ and the Wald entropy $S$;

$$
S=\frac{2 \pi}{\kappa_{n}^{2}} V_{n-2}^{(1)}\left(\frac{2 \kappa_{n}^{2} E}{(n-2) V_{n-2}^{(1)}}\right)^{(n-2) /(n-3)},
$$

Eq. (32) implies that the entropy of the black hole is equally spaced for large $E$ as

$$
S \simeq 4 \pi^{3 / 2} \hbar \frac{\Gamma\left(\frac{n-1}{2(n-3)}+\frac{1}{2}\right)}{\Gamma\left(\frac{n-1}{2(n-3)}\right)} N=: S_{N} .
$$

Some particular cases are given explicitly as

$$
S_{N}=\left\{\begin{array}{l}
4 \pi^{3 / 2} \hbar N \frac{\Gamma(2)}{\Gamma(3 / 2)}=8 \pi \hbar N \quad(n=4) \\
4 \pi^{3 / 2} \hbar N \frac{\Gamma(3 / 2)}{\Gamma(1)}=2 \pi^{2} \hbar N(n=5) \\
4 \pi^{3 / 2} \hbar \frac{\Gamma(1)}{\Gamma(1 / 2)} N=4 \pi \hbar N(n \rightarrow \infty) .
\end{array}\right.
$$

In contrast, for the asymptotically AdS black hole with general $k$, the spectrum for large $E$ is

$$
E \simeq \frac{(n-1) \hbar}{l} N
$$

where $N$ is a positive integer. The above expression becomes exact in the toroidal case $(k=0)$, which includes the BTZ black hole in three dimensions.

At a glance, two results (32) and (36) look inconsistent in the limit of vanishing cosmological constant $\Lambda \rightarrow 0$ $(l \rightarrow \infty)$ for spherical AdS black holes $(k=1)$. This is understood by noting two different length scales, the Planck length $\ell_{\mathrm{p}}:=\left(\hbar \kappa_{n}^{2} / c^{3}\right)^{1 /(n-2)}$ and the AdS radius $l$. The metric function (4) shows the relation between the mass of the black hole $E$ and the horizon radius $r_{\mathrm{h}}$ as

$$
E=\frac{(n-2) V_{n-2}^{(k)} r_{\mathrm{h}}^{n-3}}{2 \kappa_{n}^{2}}\left(k+\frac{r_{\mathrm{h}}^{2}}{l^{2}}\right) .
$$

From this relation, we see that (i) a spherical AdS black hole is approximated by an asymptotically flat black hole if $r_{\mathrm{h}} \ll l$ and (ii) it is approximated by the toroidal AdS black hole $(k=0)$ if $r_{\mathrm{h}} \gg l$. Therefore, the spectrum of the spherical AdS black hole is given by Eq. (32) in the region of $\ell_{\mathrm{p}} \ll r_{\mathrm{h}} \ll l$ and by Eq. (36) in the region of $r_{\mathrm{h}} \gg l$, as shown in Fig. 2. In the limit of vanishing cosmological constant $(l \rightarrow \infty)$, the spacetime becomes asymptotically flat so that the region $r_{\mathrm{h}} \gg l$ disappears in Fig. 2 and the mass spectrum (32) is valid in the whole region satisfying $r_{\mathrm{h}} \gg \ell_{\mathrm{p}}$.

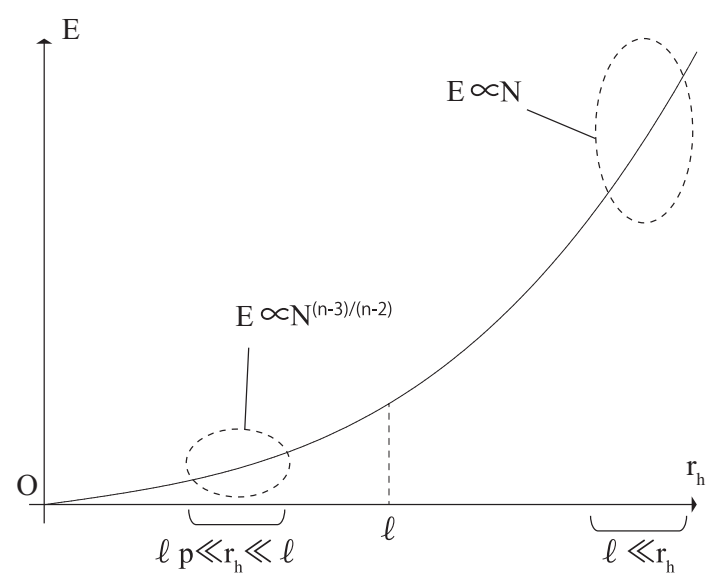

Figure 2. Mass-horizon relation (37) for spherical AdS black holes $(k=1)$. The mass spectrum is given by Eq. (32) in the region of $\ell_{\mathrm{p}} \ll r_{\mathrm{h}} \ll l$ and by Eq. (36) in the region of $r_{\mathrm{h}} \gg l$.

In summary, using the WKB approximation for the Schwarzschild-Tangherlini-type black hole (3) with large mass, we have shown that area/entropy is equally spaced in the asymptotically case, while mass is equally spaced for asymptotically AdS case. This result is unchanged under the Neumann boundary condition $(L=\infty)$. In the case of the Robin boundary condition with other values of $L$, numerical calculations are required to obtain the mass spectrum.

\section{References}

[1] J. Louko and J. Mäkelä, Phys. Rev. D 54, 4982 (1996).

[2] K. Kuchař, Phys. Rev. D50, 3961 (1994).

[3] C. W. Misner and D. H. Sharp, Phys. Rev. 136, B571 (1964).

[4] G.A.S. Dias and J.P.S. Lemos, Phys. Rev. D 79, 044013 (2009).

[5] H. Maeda and M. Nozawa, Phys. Rev. D 77, 064031 (2008).

[6] G. Kunstatter and H. Maeda, Class. Quant. Grav. 31,31, 115009 (2014). 
[7] M. Reed and B. Simon, Methods of Modern Mathematical Physics II: Fourier Analysis, Self-adjointness
(Academic Press, New York, 1975). 